\title{
Plants and Plant Products in Local Markets Within Benin City and Environs
}

\author{
Moses Edwin Osawaru and Matthew Chidozie Ogwu
}

\section{Contents}

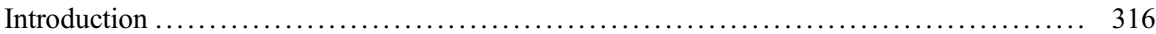

Plants and Plant Products in Local Markets: The Case of Benin City and Environs ......... 318

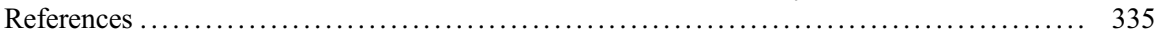

\begin{abstract}
The vulnerability of agriculture systems in Africa to climate change is directly and indirectly affecting the availability and diversity of plants and plant products available in local markets. In this chapter, markets in Benin City and environs were assessed to document the availability of plants and plant products. Markets were grouped into urban, suburban, and rural with each group having four markets. Majority of the plant and plant product vendors were women and 88 plant species belonging to 42 families were found. Their scientific and common names were documented as well as the parts of the plant and associated products available in the markets. Most of the plant and plant products found in local
\end{abstract}

This chapter was previously published non-open access with exclusive rights reserved by the Publisher. It has been changed retrospectively to open access under a CC BY 4.0 license and the copyright holder is "The Author(s)". For further details, please see the license information at the end of the chapter.

\section{E. Osawaru}

Department of Plant Biology and Biotechnology, Faculty of Life Sciences, University of Benin, Benin City, Edo State, Nigeria

M. C. Ogwu $(\bowtie)$

Department of Plant Biology and Biotechnology, Faculty of Life Sciences, University of Benin, Benin City, Edo State, Nigeria

Scuola di Bioscienze e Medicina Veterinaria, Università di Camerino - Centro Ricerche Floristiche dell'Appennino, Parco Nazionale del Gran Sasso e Monti della Laga, Barisciano (L'Aquila), Italy e-mail: matthew.ogwu@uniben.edu 
markets belong to major plant families. Urban markets had the highest diversity of plants and plant products. Three categories of plants and plant products were documented. Around $67 \%$ of the plants and plant products were categorized as whole plant/plant parts, $28 \%$ as processed plant parts, while $5 \%$ as reprocessed plant/plant parts. It was revealed that $86 \%$ of these plants are used as foods, $11 \%$ are for medicinal purposes, while $3 \%$ is used for other purposes. About $35 \%$ of plants and plant products across the markets were fruits, which is an indication that city and environs are a rich source of fruits. The local knowledge and practices associated with the plants and plant products can contribute towards formulating a strategic response for climate change impacts on agriculture, gender, poverty, food security, and plant diversity.

\section{Keywords}

Climate change $\cdot$ Ethnobotany $\cdot$ Plant diversity $\cdot$ Plant products $\cdot$ Food security $\cdot$ Market survey $\cdot$ Indigenous plants species $\cdot$ Economic plants $\cdot$ Agriculture vulnerability $\cdot$ Sustainable development

\section{Introduction}

The utilitarian nature of humans is driving the massive extinction of biodiversity, climate change, and ecological vulnerability. However, a greater understanding of plant-human interactions can contribute to sustainable development, addressing climate change and biodiversity loss, food security, and poverty reduction. All plants are considered important and can potentially serve to fulfill one or more of our basic needs - food, shelter, and clothing as well as environmental integrity. Plant product refers to goods and services derivable from plants and may include whole plant or plant part (used as ingredients and condiments). Proper local and scientific identification of plant materials is necessary to determine and predict the role of a plant and this will require a general knowledge of botany, sociology, and anthropology. Plant is essential for our continued survival on earth as they directly or indirectly provide food for survival, medicine, fibers, chemical products, and other commodities as well as to protect and maintain the environment against erosion, used to cure disease and relieve from suffering. Many industries are dependent on plants for their raw materials. Some of the most outstanding materials of modern civilization are obtained from plants, such as wood, tanning materials and dyestuffs, oils, resins, gums, varnishes, beverages, etc. Plants provide raw material for industrialization and are basis of the green revolution and a pillar for food security. The esthetic value of plants has no small influence on man's overall life satisfaction, as evidenced by the host of garden enthusiasts and flower lovers. Plants are also the basis of a vegan lifestyle.

In the economy of nature, the production and distribution of plant products have a profound influence on the environmental, economic, and social life of a nation with both domestic and international influence. The maintenance of an adequate supply of 
food and plant-based industrial raw materials is essential to the existence, as well as the prosperity, of any nation (Burkill 1985). Additionally, plants also have important roles in the tribal, social and cultural life of man (Osawaru and Dania-Ogbe 2010; Osawaru and Ogwu 2014a). Local markets are an integral part of life and cultural practice of the people especially in developing countries as a social, economic, and ecological institution. Plant products available in the market can be used as an indicator for biodiversity richness, climate changes effects, and agricultural vulnerabilities. This is more important in Africa, which according to Ogwu (2019) the environment and agriculture systems are most vulnerable climate change. Markets in rural parts of Africa are often scheduled at considerable day interval, whereas in urban and semi-urban centers, it is mostly open every day or night. Sellers have their stalls or place while hawkers also patrol the market with their various plants and plant products. Markets are rich sources of information on plants and plant products as well as an easily accessible and cost-effective place for plant-based fieldwork and germplasm collection. Markets can provide qualitative and quantitative data concerning cultural, social, and economic aspects of a plant's usage (Bye and Linares 1983; Martin 1992; Cunningham 2001). Moreover, markets are recognized as a vital botanical record of the history of useful plants in a region (Whitaker and Cutler 1966). They are places of intensive interaction between people and plants.

Local traders (mostly women) are very knowledgeable about the uses of plants and their seasonal availability. This knowledge is vital in the global response to global climate change and massive loss of biodiversity in this sixth extinction era. In Nigeria and West Africa, market vendors are known to deal in certain types of plants and plant product and are found clustered together, which imposes a sort of market influence such as fixed prices for their commodities. However, traders have certain concession such as monopoly to regular customer and slight price variations. Moreover, the survey of marketplaces provides information about food and nutritional value of plant and products as well as their ethnobotany (Nguyen 2005). Findings from such studies have been used to draw interesting conclusions and hypothesize about human-environmental-plant interactions and relationships. Climate change, migration, and economic forces can influence the availability of certain plant and plant products in the market. Obiri and Addai (2007) surveyed economic plants in Kumasi central market and documented a total of 150 plant species from 55 families most of them had multiple uses $-57 \%$ and $20 \%$ used for medicinal and food purposes, respectively. Idu et al. (2010) documented the medicinal plants sold in markets in Abeokuta, Nigeria, revealed 60 medicinal plant species used for traditional health management. The ethnobotanical survey of Yaradua and El-Ghani (2015) reported 54 plants belonging to 33 families from Katsina metropolis markets. The objective of this chapter is to identify and document the plants and plant products sold in local markets in Benin City and environs.

This chapter will compare the diversity of the plants and plant products available in local markets in urban, peri-urban, and rural centers in Benin City, Southern Nigeria. Thereafter, this chapter will categorize the plants and plant products based on the level of processing it was subjected to as well as their taxonomic families. The results will seek to promote local markets as a reservoir of plant germplasm and 
contribute towards understanding of how climate change vulnerability is affecting agriculture system in Edo state, diversity of plant and plant-based food materials available in local markets that can potentially contribute to addressing food security, poverty, and sustainable development. It will also highlight the plant parts and plant products sold in the markets.

\section{Plants and Plant Products in Local Markets: The Case of Benin City and Environs}

There are numerous open markets in Benin City (latitude $06^{\circ} 19^{\prime} 00^{\prime \prime} \mathrm{E}$ to $6^{\circ} 21^{\prime} 00^{\prime \prime} \mathrm{E}$ and longitude $5^{\circ} 34^{\prime} 00^{\prime \prime} \mathrm{E}$ to $5^{\circ} 44^{\prime} 00^{\prime \prime} \mathrm{E}$; average elevation of $77.8 \mathrm{~m}$ above sea level; 2006 est. pop. 1,147,188 with an annual growth rate of $2.9 \%$ ), which is one of the oldest cities in Nigeria and the capital of Edo State, Southern Nigeria. It is within the tropical rainforest zone of Nigeria with an estimated area of $550 \mathrm{~km}^{2}$. Geologically the city has a sedimentary formation of the Miocene-Pleistocene age. Benin has an undulating topography with a vegetation type characterized by lowland rainforest and an annual average rainfall of $800 \mathrm{~mm}$. A 35-year study by Floyd et al. (2016) revealed massive climate fluctuations especially in average rainfall, temperate, humidity and suggested that it is impacting plant production and environmental changes due to soil erosion. The fluctuation is attributed to high anthropogenic activities in Benin City (Efe and Eyefia 2014). With increased warming, flooding, and urbanization, agriculture and food production are threatened in Benin City (Atedhor et al. 2011).

Benin is the center of Nigeria's rubber industry, but processing palm nuts for oil is also an important traditional industry. Benin has numerous local markets strewn across the city to cater to the needs of its inhabitants as well as to serve as a sales outlet for the numerous farm produce cultivated in the rural areas of the state as well as in urban home gardens. The nodal nature of Benin makes it an ideal place for various commercial activities as these farm produce can be easily transported to cities like Lagos, Abuja, and Port Harcourt. Benin City is endowed with a wide diversity of plants and plant products. The sales of plants and plant products play a key role in the sustenance of livelihoods of people providing income, employment, food, and medicines among others in Benin City and environs. Some of the local markets in Benin City and environs are God's Market (Ekiosa), Oba Market (Ekioba), New Benin Market, Santana Market, Uselu Market (Ediaken Market), Oliha Market, Ugbogiobo Market, Evbuotubu Market, Oregbeni Market (Ikpoba Hill Market), Ekiadolor Market, Iguobazuwa Market, Ehor Market, and Usen Market.

The sampling frame considered markets within Benin City and environs, which were delimited into three categories according to the status defined by Osawaru and Odin (2012) - urban, peri-urban, and rural. A reconnaissance visit was undertaken to all the local markets in Benin City and environs. Twelve markets were randomly selected for sampling. They consist of four urban, four peri-urban, and four rural markets (Table 1). 
Table 1 Sampling sites for the survey of plants and plant products in Benin City and environs

\begin{tabular}{l|l|l}
\hline Market & Category & Local government area \\
\hline New Benin & Urban & Oredo \\
\hline Uselu & Urban & Egor \\
\hline Oba & Urban & Oredo \\
\hline Oregbene & Urban & Ikpoba-Okha \\
\hline Evbuotubu & Peri-urban & Egor \\
\hline Ugbogiobo & Peri-urban & Ovia North East \\
\hline Ugbiohioko & Peri urban & Egor \\
\hline Iguobazuwa & Peri-urban & Ovia South West \\
\hline Ehor & Rural & Uhunnwode \\
\hline Usen & Rural & Ovia North East \\
\hline Ekiadolor & Rural & Ovia North East \\
\hline Ugbogui & Rural & Ovia South West \\
\hline
\end{tabular}

In each market, ten traders of mixed age and sex were randomly selected and plants and plant products in their stalls were assessed. Each market was visited three times. First, to map out the randomly selected informant, secondly, to administer the questionnaire and inventory the plants and plant products, and finally, to seek clarity for some questions outlined in the questionnaire. Responses via the questionnaires were retrieved from the questionnaires, translated and scored by typing into Microsoft Excel, and analyzed quantitatively. Plants and plant products were categorized according to Osawaru and Odin (2012) i.e.,

1. $1^{0}$ of plants and plant products-whole plant/plant part

2. $2^{0}$ of plant and plant product-processed plant part

3. $3^{0}$ of plant and plant product-reprocessed plant/plant part

Majority of the traders encountered in the markets were women. This confirms the findings of De Caluwe (2011) and Agea et al. (2011) that trading in plant and plant products are dominated by women. In a different study, Osawaru and Ogwu (2014b) also established that women contribute significantly to holding plant germplasm. These findings underscore the importance of women in the fight to address the effects of climate change especially food security and sustainable agriculture. Moreover, the sales of plants and plant products in the different markets were practiced by different tribes and ethnic groups in all the markets surveyed.

A total of 88 plants and plants product was found in all the local markets assessed (Table 2). The botanical and common names, forms, diversity, and categories of the plant and plant products sold in local markets within Benin City and environs are presented in Table 2. These 88 plants and plant product are distributed into 42 families. Presence of plant and plant products varies in the different markets. For instance, Adansonia digitata, Brassica oleracea, Cucumis sativus, Cucurbita pepo, Cyperus esculetus, Dialium guineense, Ricinus communis, Pennisetum glaucum, Pentaclathra macrophylla, Myristica fragans, and Phoenix dactylifera 
Table 2 Diversity of plants and plant products in 12 local markets within Benin City and environs

\begin{tabular}{|c|c|c|c|c|c|c|}
\hline \multirow[b]{2}{*}{ Botanical name } & \multirow[b]{2}{*}{ Family } & \multirow{2}{*}{$\begin{array}{l}\text { Common } \\
\text { name }\end{array}$} & \multirow{2}{*}{$\begin{array}{l}\text { Form/product } \\
\text { type }\end{array}$} & \multirow{2}{*}{$\begin{array}{l}\text { Local name } \\
\text { (Bini) }\end{array}$} & \multicolumn{2}{|c|}{ Urban market } \\
\hline & & & & & New Benin & Uselu \\
\hline Abelmoshus esculentus L. & Malvaceae & Okra & & Ikhiav-bo & + & + \\
\hline Adensonia digitata $\mathrm{L}$. & Malvaceae & Baobab & & & + & + \\
\hline Allium cepa $\mathrm{L}$. & Liliaceae & Onion & & Alubara & + & + \\
\hline Allium sativum $\mathrm{L}$. & Liliaceae & Garlic & & & + & + \\
\hline Amaranthus caudatus $\mathrm{L}$. & Amaranthaceae & Spinach & & Ebaafor & + & + \\
\hline Anacardium occidentalis L. & Anacadiaceae & Cashew & & & + & - \\
\hline Ananas comosus L. & Bromeliaceae & Pineapple & & Edinebo & + & + \\
\hline Annona muricata L. & Annonaceae & Soursop & & & + & + \\
\hline Arachis hypogaea L. & Fabaceae & Groundnut & & Isaerewe & + & + \\
\hline Azadirachta indica $\mathrm{L}$. & Meliaceae & Neem & & & - & + \\
\hline Bombax buonopozense L. & Malvaceae & & & & - & - \\
\hline Brassica oleracea L. & Brassicaceae & Cabbage & & & + & + \\
\hline Calotropis.procera Auton. & Apocynaceae & & & & + & + \\
\hline Capsicum annum $\mathrm{L}$. & Solanaceae & Pepper & & Ehien & + & + \\
\hline $\begin{array}{l}\text { Capsicum } \\
\text { frutescens } \mathrm{L} .\end{array}$ & Solanaceae & Pepper & & Ikpovb-ukho & + & + \\
\hline Carica papaya $\mathrm{L}$. & Caricaeae & Pawpaw & & Uhoro & + & + \\
\hline Celosia argentea $\mathrm{L}$. & Amaranthaceae & Celosia & & & + & + \\
\hline $\begin{array}{l}\text { Citrus } \\
\text { aurantifolia } \mathrm{L} .\end{array}$ & Rutaceae & Lime & & Alimonegiere & + & + \\
\hline Citrus limon $\mathrm{L}$. & Rutaceae & Lemon & & & + & + \\
\hline Citrus sinensis Osbeck & Rutaceae & Orange & & Alimebo & + & + \\
\hline Cochorus olitorius L. & Tiliaceae & Jute & & & + & + \\
\hline Cocos nucifera $\mathrm{L}$. & Palmae & Coconut & & Ivin & + & + \\
\hline Cola acuminate Engl. & Sterculiaceae & Kolanuts & & Gbanja & + & + \\
\hline Cola nitida Schum. & Sterculiaceae & Kolanut & & Evbedo & + & + \\
\hline Colocasia esculenta Schott & Araceae & Cocoyam & & Akaha & + & + \\
\hline Crescentia cujele L. & Curcubitaceae & Calabash & & Uko & + & + \\
\hline $\begin{array}{l}\text { Cucumeropsis mannii } \\
\text { Naudin }\end{array}$ & Cucurbitaceae & Melon & & Ogi & + & + \\
\hline Cucumis sativus L. & Cucurbitaceae & Cucumber & & & + & + \\
\hline Cucurbita pepo L. & Cucurbitaceae & & & & + & + \\
\hline Cucurma longa $\mathrm{L}$. & Zingiberaceae & & & & + & + \\
\hline $\begin{array}{l}\text { Cymbopogon } \\
\text { citrates L. }\end{array}$ & Poaceae & $\begin{array}{l}\text { Lemmon } \\
\text { grass }\end{array}$ & & Ebiti & + & + \\
\hline Cyperus esculentus $\mathrm{L}$. & Cyperaceae & Tiger nut & & & + & + \\
\hline Dacryodes edulis Lam & Burderaceae & African pear & & Oruvbu & - & - \\
\hline Daucus carota L. & Apiaceae & Carrot & & & + & + \\
\hline $\begin{array}{l}\text { Dennettia tripetala } \\
\text { Bak. F. }\end{array}$ & Annonaceae & Pepper fruit & & Ako & + & + \\
\hline Dialium guineense Willd & Fabaceae & $\begin{array}{l}\text { Velvet } \\
\text { tamarind }\end{array}$ & & & + & + \\
\hline Dioscorea alata Lour & Dioscoreaceae & Water yam & & Igierua & + & + \\
\hline Dioscorea cayenensis Lam. & Dioscoreaceae & Aerial yam & & Ikpen & + & + \\
\hline
\end{tabular}




\begin{tabular}{|c|c|c|c|c|c|c|c|c|c|}
\hline \multirow[b]{2}{*}{ Oba } & \multirow[b]{2}{*}{ Oregbene } & \multicolumn{4}{|c|}{ Peri-urban market } & \multicolumn{4}{|c|}{ Rural market } \\
\hline & & Evbuotubu & Ugbogiobo & Ugbiohioko & Iguobazuwa & Ehor & Usen & Ekiadolor & Ugbogui \\
\hline+ & + & + & + & + & + & + & + & + & + \\
\hline+ & + & + & - & - & - & - & + & + & - \\
\hline+ & + & + & + & + & + & + & + & + & + \\
\hline+ & + & + & + & + & + & + & + & + & + \\
\hline+ & + & + & + & + & + & + & + & + & + \\
\hline+ & - & + & + & - & + & + & + & + & + \\
\hline+ & + & + & + & + & + & + & + & + & + \\
\hline+ & + & + & - & - & - & - & - & + & + \\
\hline+ & + & + & + & + & + & + & + & + & + \\
\hline+ & - & + & + & + & + & + & - & - & - \\
\hline- & - & + & + & + & + & - & + & + & + \\
\hline+ & + & + & - & + & - & - & - & - & - \\
\hline+ & - & - & - & - & + & - & + & - & - \\
\hline+ & + & + & + & + & + & + & + & + & + \\
\hline+ & + & + & + & - & - & - & - & - & - \\
\hline+ & + & + & + & + & + & + & + & + & + \\
\hline+ & + & + & + & + & + & + & + & + & + \\
\hline+ & + & + & + & + & + & + & + & + & + \\
\hline+ & + & + & + & - & - & - & + & + & - \\
\hline+ & + & + & + & + & + & + & + & + & + \\
\hline+ & + & + & + & + & + & + & + & + & + \\
\hline+ & + & + & + & + & + & + & + & + & + \\
\hline+ & + & + & + & + & + & + & + & + & + \\
\hline+ & + & + & + & + & + & + & + & + & + \\
\hline+ & + & + & + & + & + & + & + & + & + \\
\hline+ & + & - & + & + & + & + & - & - & - \\
\hline+ & + & + & + & + & + & + & + & + & + \\
\hline+ & + & + & + & - & - & - & - & + & - \\
\hline+ & + & + & + & - & - & + & - & - & - \\
\hline+ & + & - & - & + & - & - & - & - & - \\
\hline+ & + & + & - & - & - & + & - & - & + \\
\hline+ & + & - & - & - & - & - & - & - & - \\
\hline- & - & - & + & + & + & + & + & + & - \\
\hline+ & + & + & + & + & + & - & - & + & - \\
\hline- & + & - & + & + & - & + & + & - & + \\
\hline+ & + & - & + & + & + & - & - & - & - \\
\hline+ & + & + & + & + & + & + & + & + & + \\
\hline+ & + & + & + & + & + & + & + & + & + \\
\hline
\end{tabular}


Table 2 (continued)

\begin{tabular}{|c|c|c|c|c|c|c|}
\hline \multirow[b]{2}{*}{ Botanical name } & \multirow[b]{2}{*}{ Family } & \multirow{2}{*}{$\begin{array}{l}\text { Common } \\
\text { name }\end{array}$} & \multirow{2}{*}{$\begin{array}{l}\text { Form/product } \\
\text { type }\end{array}$} & \multirow{2}{*}{$\begin{array}{l}\text { Local name } \\
\text { (Bini) }\end{array}$} & \multicolumn{2}{|c|}{ Urban market } \\
\hline & & & & & New Benin & Uselu \\
\hline Dioscorea rotundata Poir & Dioscoreaceae & Yam & $\begin{array}{l}\text { Yam chips } \\
\text { and yam flour }\end{array}$ & Emowe & + & + \\
\hline Elaeis guineensis Jacq & Palmae & Oil palm & Oil & Udin & + & + \\
\hline Garcina cola Heckel & Guittiferae & Bitter cola & & Edun & + & + \\
\hline Glycine $\max \mathrm{L}$. & Fabaceae & Soy bean & & Owerie-otan & + & + \\
\hline Gnetum africanum Welw. & Gnetaceae & & & & + & + \\
\hline Gossypium hirsutum L. & Malvaceae & Cotton & & Oruhu & + & + \\
\hline Hibiscus cannabinus L. & Malvaceae & & & & + & + \\
\hline Hibiscus sabdarifa L. & Malvaceae & Roselle & & Zobo & + & + \\
\hline Ipomea batata L. & Convolvulaceae & $\begin{array}{l}\text { Sweet } \\
\text { potato }\end{array}$ & & Iyinebo & + & + \\
\hline Irvingia gabonensis Baill & Irvingiaceae & Bush mango & & Ogwi & + & + \\
\hline $\begin{array}{l}\text { Lycopersicum esculentum } \\
\text { L. }\end{array}$ & Solanaceae & Tomato & Tomato paste & Etomat-osi & + & + \\
\hline Mangifera indica L. & Anacadiaceae & Mango & & Emango & + & + \\
\hline Manihot esculatua Crantz & Euphobiaceae & Cassava & $\begin{array}{l}\text { Garri, fufu, } \\
\text { bobozi and } \\
\text { cassava flour }\end{array}$ & Igari & + & + \\
\hline Murraya koenigii L. & Rutaceae & Curry & & Curry leaf & + & + \\
\hline Musa paradisiaca L. & Musaceae & Banana & & & + & + \\
\hline Musa sapientum Linn & Musaceae & Plantain & $\begin{array}{l}\text { Chips and } \\
\text { plantain flour }\end{array}$ & Oghede & + & + \\
\hline Myristica fragrans Houtt. & Myristicaceae & Nutmeg & & & + & + \\
\hline Ocimum gratissimum Linn & Lasiottae & Scent leaf & & Ebihiri & + & + \\
\hline Oryza sativa L. & Poaceae & Rice & & Izee & + & + \\
\hline $\begin{array}{l}\text { Parkia clappertoniana } \\
\text { Keay }\end{array}$ & Fabaceae & Locust bean & & Evbarie & + & + \\
\hline $\begin{array}{l}\text { Pennisetum glaucum (L.) R. } \\
\text { Br. }\end{array}$ & Poaceae & Millet & Kunu & & + & + \\
\hline $\begin{array}{l}\text { Pentaclethra macrophylla } \\
\text { L. }\end{array}$ & Fabaceae & $\begin{array}{l}\text { African oil } \\
\text { bean }\end{array}$ & & & + & + \\
\hline Persea americana Miller & Lauraceae & $\begin{array}{l}\text { Avocado } \\
\text { Pear }\end{array}$ & & & + & + \\
\hline Phaseolus vulgaris L. & Fabaceae & Beans & $\begin{array}{l}\text { Beans cake, } \\
\text { beans flour }\end{array}$ & Ere & + & + \\
\hline Phoenix dactylifera $\mathrm{L}$. & Palmae & Date palm & & & + & + \\
\hline Piper guineense Schumach & Piperaceae & $\begin{array}{l}\text { African } \\
\text { pepper }\end{array}$ & & Oziza & + & + \\
\hline Psidium guajava L. & Myrtaceae & Guava & & & - & - \\
\hline Rauwolfia vomitoria Afzel. & Apocynaceae & Rauwolfia & & Akata & - & + \\
\hline Ricinus communis L. & Euphorbiaceae & Castor oil & & & + & + \\
\hline Saccharum officinarum L. & Solanaceae & Sugar cane & Sugar & Ukhure & + & + \\
\hline Sesanum orientale L. & Pedaliacaece & & & & + & + \\
\hline Solanum melogena L. & Convolvulaceae & Garden-egg & & Ekhue & + & + \\
\hline Solanum tuberosum L. & Poaceae & Irish potato & & & + & + \\
\hline Sorghum bicolour L. & Poaceae & Guinea corn & Kunu & & + & + \\
\hline Spondias mombin L. & Meliaceae & Hug phem & & Okhikhan & - & + \\
\hline
\end{tabular}




\begin{tabular}{|c|c|c|c|c|c|c|c|c|c|}
\hline \multirow[b]{2}{*}{ Oba } & \multirow[b]{2}{*}{ Oregbene } & \multicolumn{4}{|c|}{ Peri-urban market } & \multicolumn{4}{|c|}{ Rural market } \\
\hline & & Evbuotubu & Ugbogiobo & Ugbiohioko & Iguobazuwa & Ehor & Usen & Ekiadolor & Ugbogui \\
\hline+ & + & + & + & + & + & + & + & + & + \\
\hline+ & + & + & + & + & + & + & + & + & + \\
\hline+ & + & + & + & - & + & - & + & - & + \\
\hline+ & + & + & + & + & + & + & + & + & + \\
\hline+ & + & + & - & - & + & + & + & - & + \\
\hline+ & + & + & + & + & + & + & + & - & + \\
\hline+ & - & - & - & - & - & + & - & + & - \\
\hline+ & + & + & + & + & - & - & + & - & + \\
\hline+ & + & + & + & + & + & + & + & + & + \\
\hline+ & + & + & + & + & + & + & + & + & + \\
\hline+ & + & + & + & + & + & + & + & + & + \\
\hline+ & + & + & + & + & + & + & + & + & + \\
\hline+ & + & + & + & + & + & + & + & + & + \\
\hline+ & + & + & + & + & + & + & + & + & + \\
\hline+ & + & + & + & + & + & + & + & + & + \\
\hline+ & + & + & + & + & + & + & + & + & + \\
\hline+ & + & + & - & + & - & - & - & - & - \\
\hline+ & + & + & + & + & + & + & + & + & + \\
\hline+ & + & + & + & + & + & + & + & + & + \\
\hline+ & + & + & + & + & + & + & + & + & + \\
\hline+ & + & - & - & - & - & - & - & - & - \\
\hline+ & + & - & - & - & - & - & - & - & - \\
\hline+ & + & - & + & + & + & - & - & - & + \\
\hline+ & + & + & + & + & + & + & + & + & + \\
\hline+ & - & - & - & - & - & - & - & - & - \\
\hline+ & + & - & - & - & + & + & - & + & - \\
\hline+ & + & + & + & + & - & - & - & - & - \\
\hline+ & + & - & - & - & - & + & + & + & + \\
\hline+ & - & - & - & - & - & - & - & - & - \\
\hline+ & + & + & - & + & + & - & - & - & + \\
\hline+ & + & + & + & + & - & - & - & - & - \\
\hline+ & + & + & + & + & + & + & + & + & + \\
\hline+ & + & + & - & + & - & - & - & - & - \\
\hline+ & - & - & - & - & - & - & - & - & - \\
\hline+ & + & + & + & + & - & - & - & - & - \\
\hline
\end{tabular}


Table 2 (continued)

\begin{tabular}{|c|c|c|c|c|c|c|}
\hline \multirow[b]{2}{*}{ Botanical name } & \multirow[b]{2}{*}{ Family } & \multirow{2}{*}{$\begin{array}{l}\text { Common } \\
\text { name }\end{array}$} & \multirow{2}{*}{$\begin{array}{l}\text { Form/product } \\
\text { type }\end{array}$} & \multirow{2}{*}{$\begin{array}{l}\text { Local name } \\
\text { (Bini) }\end{array}$} & \multicolumn{2}{|c|}{ Urban market } \\
\hline & & & & & New Benin & Uselu \\
\hline Talfairia occidentalis Hook & Cucurbitaceae & Pumpkin & & Uvbeg-hen & + & + \\
\hline Talinum triangulare Jacq & Portulaceae & Water leaf & & Ebodo-don & + & + \\
\hline Tamarindus indica $\mathrm{L}$. & Fabeaceae & & & & + & + \\
\hline $\begin{array}{l}\text { Tetrochidium } \\
\text { didymostemon } \\
\text { (Baill.) Pax \& K. Hoffm }\end{array}$ & Euphorbiaceae & & & & - & - \\
\hline $\begin{array}{l}\text { Thaumatococcus danielli } \\
\text { Benth. }\end{array}$ & Marantaceae & & & Ebe-eba & + & + \\
\hline Theobroma cacao L. & Sterculiaceae & Cocoa & & Koko & + & - \\
\hline Thymus vulgaris L. & Lamiaceae & Thyme & & & + & + \\
\hline Treculia Africana Decne. & Moraceae & $\begin{array}{l}\text { African } \\
\text { breadfruit }\end{array}$ & & & + & + \\
\hline $\begin{array}{l}\text { Trilepisium } \\
\text { madagascariensis } \\
\text { DC }\end{array}$ & Apocynaceae & & & & + & + \\
\hline Triticum aestivum $\mathrm{L}$. & Poaceae & $\begin{array}{l}\text { Bread } \\
\text { (processed } \\
\text { wheat) }\end{array}$ & & & + & + \\
\hline $\begin{array}{l}\text { Vernonia amygdalina } \\
\text { Delile }\end{array}$ & Asteraceae & Bitter leaf & & Oriwo & + & + \\
\hline Vigna unguiculata $\mathrm{L}$. & Fabaceae & Cow pea & & Ere & + & + \\
\hline Vitellaria paradoxa Gaertn & Sapotaceae & Shea butter & & & + & + \\
\hline $\begin{array}{l}\text { Xylopia aethiopica (Dunal) } \\
\text { A. Rich }\end{array}$ & Lauraceae & & & Unie & - & - \\
\hline Zea mays $\mathrm{L}$. & Poaceae & Maize & Corn flour & Okha & + & + \\
\hline Zingiber officinale Roscoe & Zingibeaceae & Ginger & & & + & + \\
\hline
\end{tabular}

$+=$ Present $;-=$ Absent 


\begin{tabular}{|c|c|c|c|c|c|c|c|c|c|}
\hline & & Peri-urban $n$ & arket & & & Rural & narket & & \\
\hline Oba & Oregbene & Evbuotubu & Ugbogiobo & Ugbiohioko & Iguobazuwa & Ehor & Usen & Ekiadolor & Ugbogui \\
\hline+ & + & + & + & + & + & + & + & + & + \\
\hline+ & + & + & + & + & + & + & + & + & + \\
\hline+ & - & + & - & - & - & - & - & - & + \\
\hline- & - & + & + & + & + & + & + & + & + \\
\hline+ & + & + & + & + & + & + & + & + & + \\
\hline+ & - & - & - & + & + & + & - & - & + \\
\hline+ & + & + & + & + & + & + & - & + & + \\
\hline- & - & - & - & - & - & - & + & + & + \\
\hline+ & - & - & - & - & - & + & - & + & - \\
\hline+ & + & + & + & + & + & + & + & + & + \\
\hline+ & + & + & + & + & + & + & + & + & + \\
\hline+ & + & + & + & + & + & + & + & + & + \\
\hline+ & + & + & + & + & + & + & + & + & + \\
\hline- & - & - & + & + & + & + & + & + & - \\
\hline+ & + & + & + & + & + & + & + & + & + \\
\hline+ & + & + & + & + & + & + & + & + & + \\
\hline
\end{tabular}


were more common in urban markets than in rural markets. On the other hand, Tetrochidium didymostemon, Xylopia aethiopica, Anacardium occidentalis, Bombax buonopozense, and Dacryodes edulis were found mostly in rural markets. However, this trend might not translate directly into plant diversity in urban and rural centers but their utilization patterns. In Benin City, peri-urban markets mostly act as a transition zone for rural and urban markets. Previous investigations of rural markets in Nigeria by Johnson and Johnson (1976) recorded 58 species of plants sold in Nigeria, Keratela and Hussain (1990) reported 21 species, Gill et al. (1993) recorded 93 plants, Idu et al. (2010) reported 103 and Osawaru and Odin (2012) reported 117. The difference in the number of plants recorded from the different study might be related to the season when the study was undertaken as well as the agricultural yield of the previous seasons and change in attitude, taste, where vendors source their plants, prevailing economic and environmental conditions. Mekasha and Tirfe (2019) highlighted that the marketing of agricultural produce requires planned production, grading of products, transportation to markets, distribution, pricing, and advertisement. Most of the plant species found in the markets are exotic. This supports the report of Muhanji et al. (2011) and Ogwu et al. (2016, 2017) which opined that the colonial era introduced and promoted the production and sale of plants exotic to Africa. Overall, the plants and plant products distribution across the market ranged from 58 to 83 in all the markets assessed (Fig. 1). There are more plants and plant products in urban markets compared to peri-urban and rural markets. This might be due to the higher food demands of the growing urban population, urbanization policies, greater economic power, and migration (Romanik 2008; Ogwu 2019). If the current urbanization trend is left unchecked, it might increase the vulnerability of African cities to climate change as well as challenges associated with food security. Another reason for the high distribution of plant species in urban markets might be the large size of these markets and the age-long attitude of rural dwellers to bring their farm produce to city centers for sale. The least diversity was in Usen Market while Uselu Market had the highest species composition. Overall, the urban markets had higher species composition. Next to the urban markets were peri-urban markets in plant and plant product abundance.

Plant products found in the different markets were assessed based on the level of processing that has been done to the plants. The classification of plant processing included first, second, and third-degree of plant and plant products (Fig. 2). The first degree of plant and plant products refers to the whole plant or plant part, while the second degree of plant and plant products and third degree of plant and plant products are processed and reprocessed plant or plant parts, respectively. It was also observed that most plant products were only processed once before been presented for sale in the markets (Fig. 2).

Plants and plant products in the local markets can be grouped into cereals (e.g., maize, rice, guinea corn, millet etc.), legumes (e.g., beans, groundnut, soybean etc.), stem tubers (e.g., yam and Irish potato), root tubers (e.g., cassava, carrot and sweet potato), fruits (e.g., pawpaw, orange, pineapple, mango, banana, pear, etc.), vegetables (e.g., waterleaf, bitter leaf, Amaranthus sp., Celosia sp., pumpkin leaf etc.), nuts (e.g., coconut), oil (e.g., palm fruit), spices (e.g., pepper, onion, ginger, garlic etc.). 


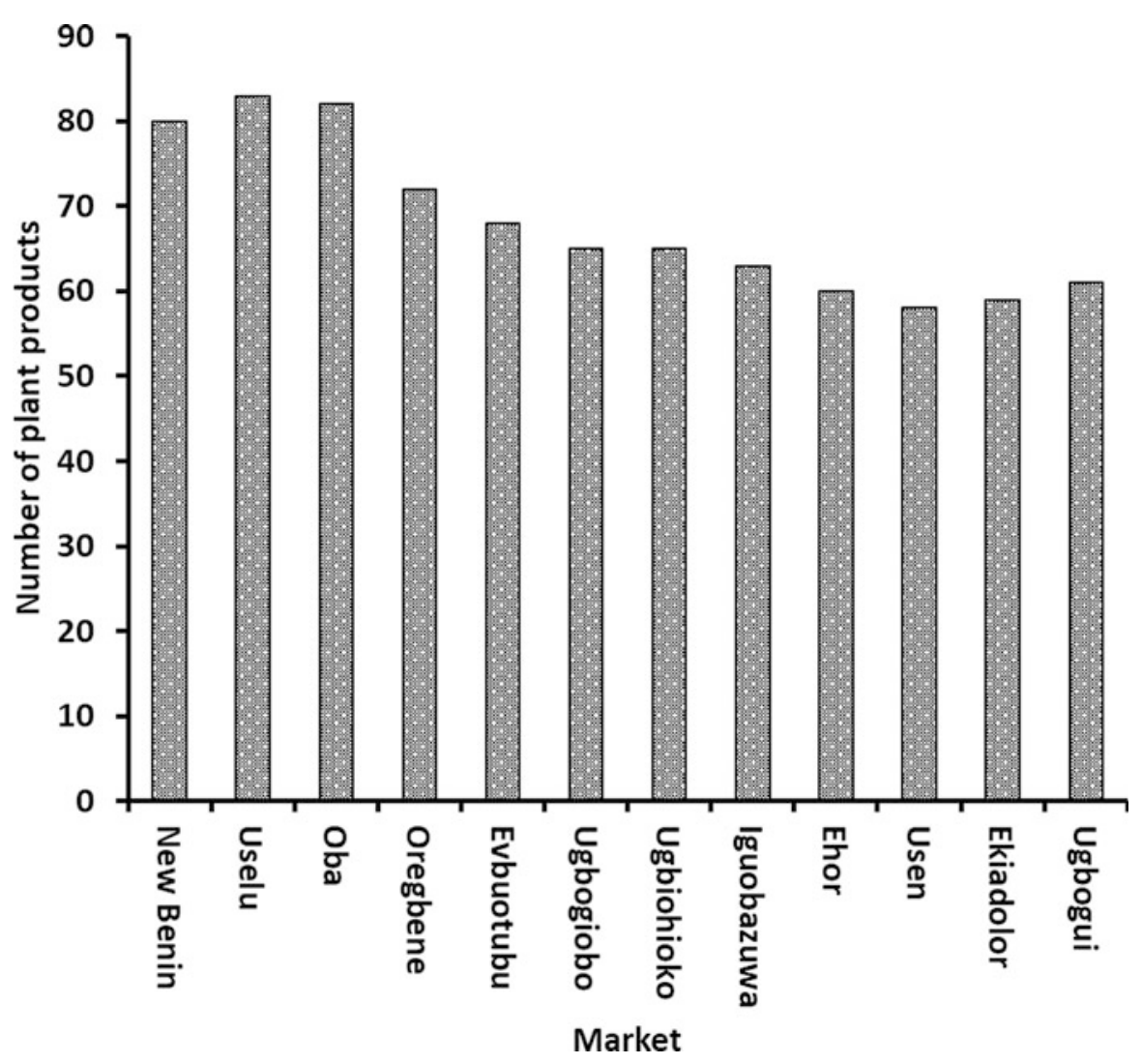

Fig. 1 Number of plants and plant products in local markets in Benin City and environs

Fig. 2 Category of plants based on forms in which they are available in the market

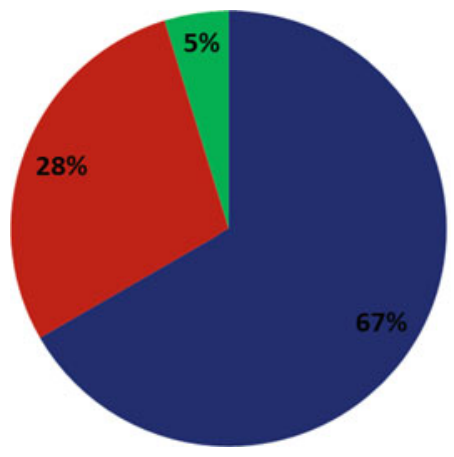

- First degree of plants and plant products

necond degree of plant and plant products

" Third degree of plant and plant product

The dominance of first degree processed plant products could be attributed to the near lack of government support for the agriculture sector of Nigeria. It also suggests Nigerians might prefer plant products that have undergone little to no processing. It 
was observed that the availability of plants and their products rely on seasonal variations. The plants and plant products were seen in different forms in the markets depending on the season. Markets are made up $90-99 \%$ of plants and plant products. Some of these products may have been to the $3 \mathrm{rd}$ degree of processing, for example, Rain boot, etc. However, not all part of the plant may be essential. These forms are fruits, leaves, rhizomes, bulbs, corms, stem tuber, root tuber and also in processed forms such as fufu, gari, and cassava flour from cassava; oil, broom, and basket from oil palm; tomato paste from tomato, etc. These different forms are the status in which these plants and plant products are best sold and preserved. This is in line with the report of Idu et al. (2005).

The utilization pattern of the plants and plant products is presented in Table 3. Major categories included cereals, legumes, roots and tubers, fruits, fats and oils, sugar crops, fiber crops, spice and condiments, beverages, medicinal, and others. It was observed that $86 \%$ of the plants and plant products are used for foods, $10 \%$ are used for medicinal purpose while 3\% is used for other purposes. However, the highest percentage was noticed in fruits, which are $35 \%$.

The habit of plants found in local markets in Benin City and environs range from grasses, herbs, shrubs, and trees to vines. Overall, the composition was $39 \%, 20 \%$, $17 \%, 15 \%$, and $9 \%$ for trees, shrubs, grasses, herbs, and vines, respectively (Fig. 3). This is an indication that for plants and plant products gotten from tree crops, buyers, and vendors will have to wait for months or years before parts that are available to be harvested for sale or consumption.

The plant species recorded in the study are mainly used as a source of food, cash or medicine. Others supply diversity, essential nutrients, vitamins, or minerals in diets that would otherwise consist primarily of carbohydrates (Johns 2004; Johns and Sthapit 2004). Our investigation revealed that most of the plants and plant products are mainly for foods while a few are for medicinal and other uses. Often, they reflect cultural values and as a pool of health and nutritional information for the public and health practitioners (Johns and Eyzaguirre 2002). The availability of diverse plant and plant products in the market relies on local agriculture system, seasonal variations, local knowledge and practices, as well as plant germplasm. Produce are mainly sourced locally from home gardens and distant farms. Therefore, the vulnerability of agriculture systems in Benin City to climate change might not be affecting the production of the plants reported in this chapter in the short term. However, the interplay of diverse external factors and climate change is likely to affect the availability of plant and plant products currently found in open markets in the long term. It is recommended that the roles of women, soil health, and plant diversity be assessed in order to formulate a policy to mitigate and adapt to climate change impact on agriculture and food security in Benin City and other parts of Nigeria and Africa.

Moreover, considering that Muhanji et al. (2011) reported that there might be 45,000 plant species in Africa, the amount of plant and plant products recorded in open markets in Benin City only represent a small portion of that diversity. About $86 \%$ of the plants and plant products found in the markets in Benin City and environs are used for foods, $11 \%$ are for medicinal purpose, while $3 \%$ are used for others 


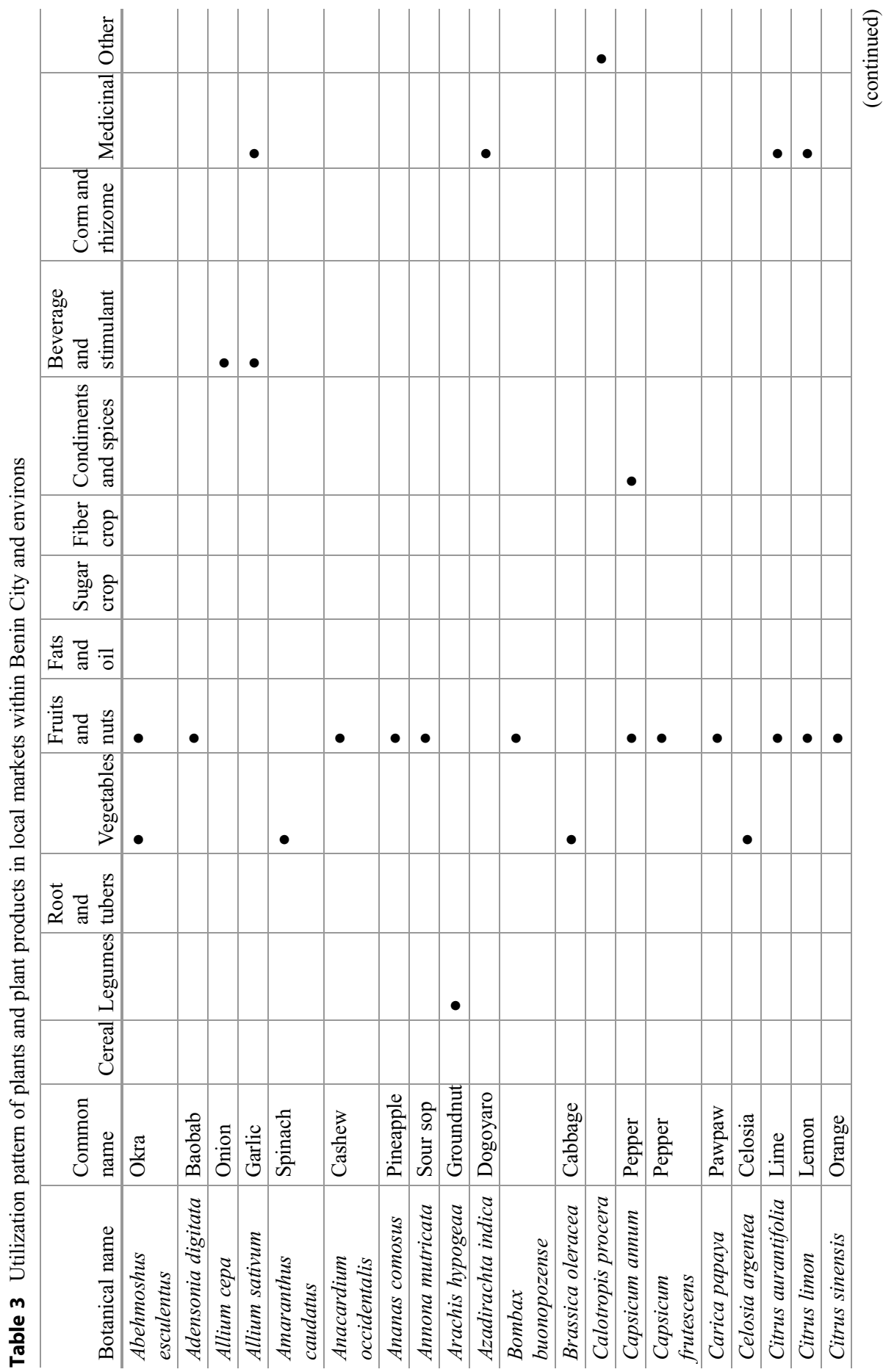




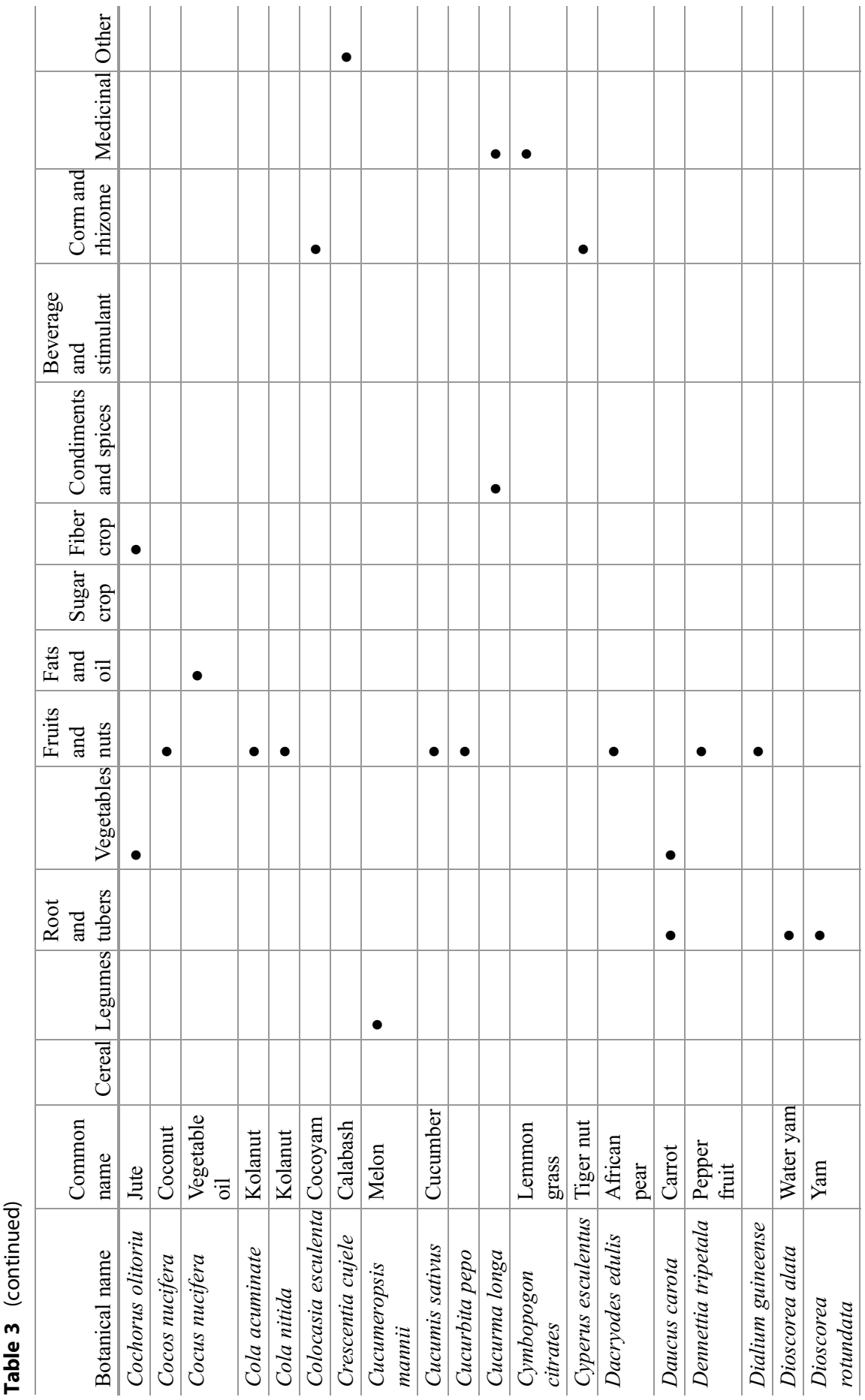




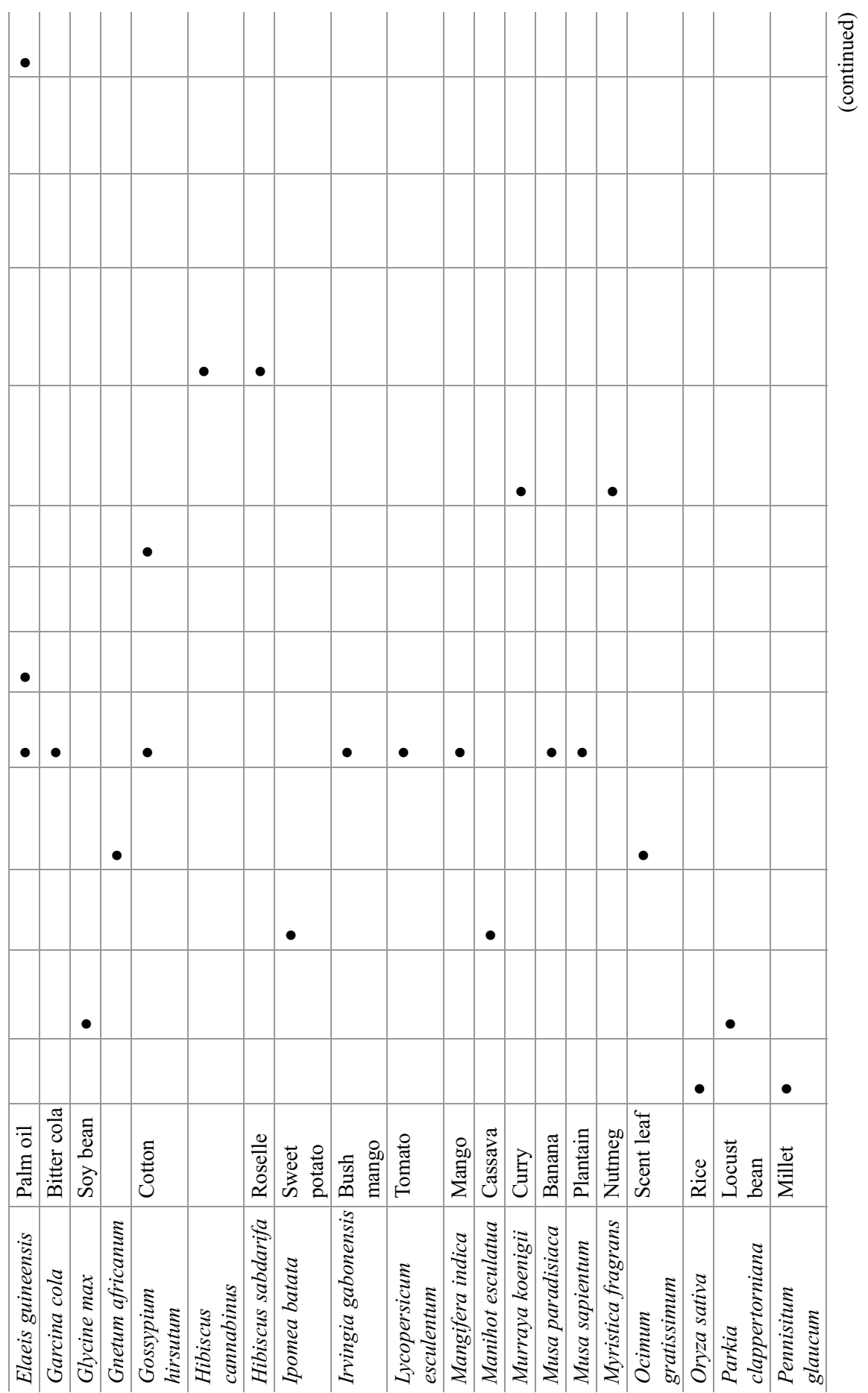




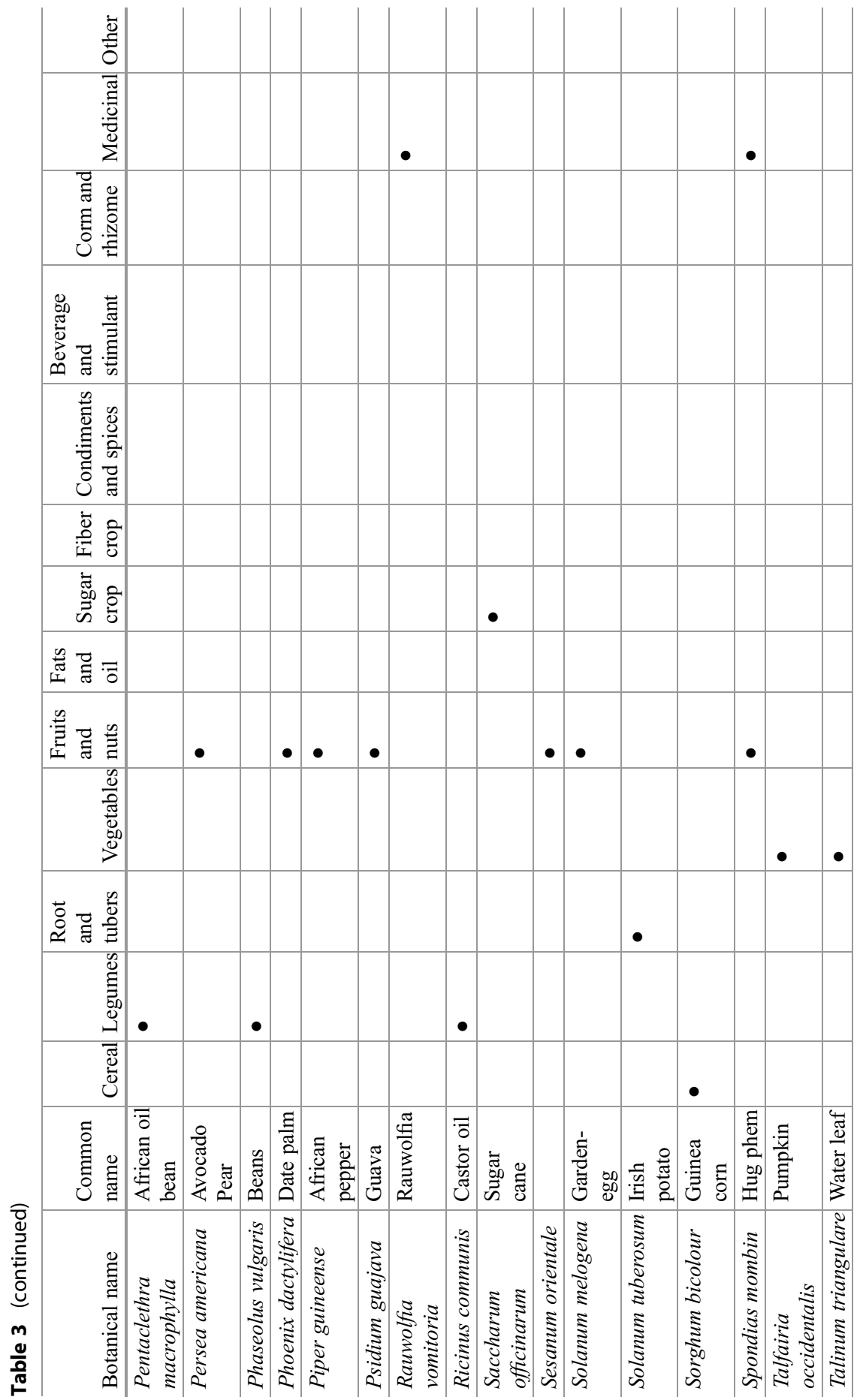




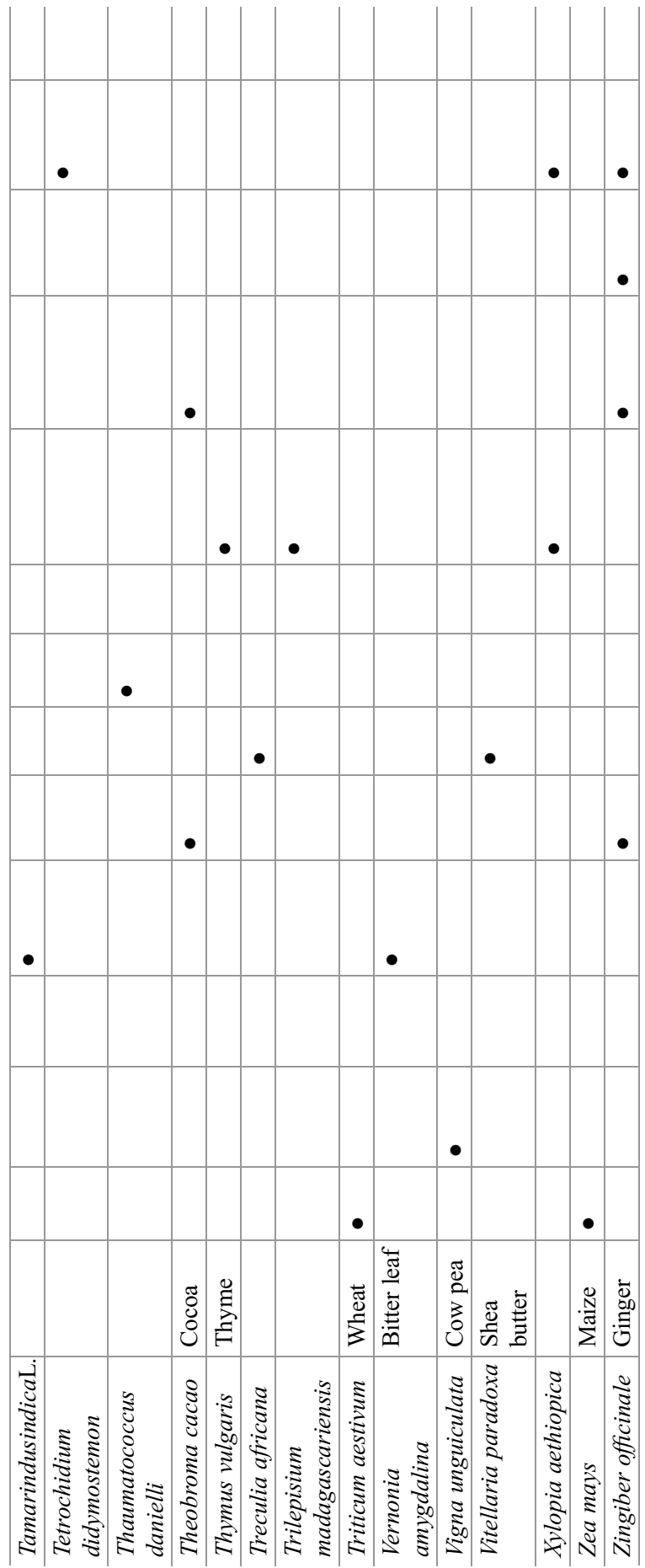


Fig. 3 The habit of plants and plant products in local markets within Benin City and environs

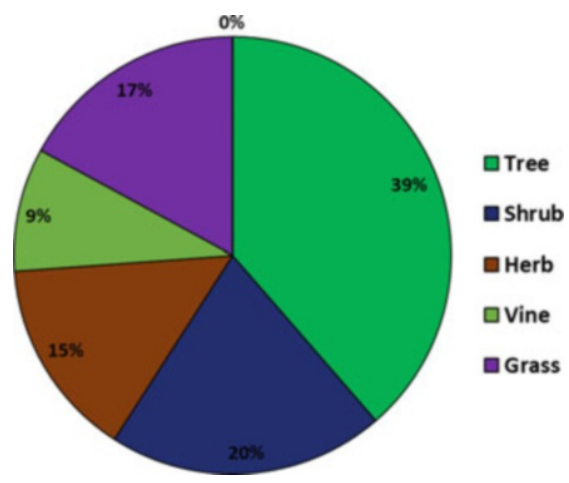

purpose. Of the $86 \%$ used for food purpose, fruits and vegetable had the highest percentage of usage, which are $35 \%$ and $9 \%$ for fruits and vegetables, respectively. This is an indication that the markets in Benin City and environs have rich and diverse pool of fruits and vegetables, which are of great nutritional value. This is in line with the study of Odhav et al. (2007) and Ogwu et al. (2016) wherein they pointed out that indigenous vegetables and fruits represent inexpensive but high quality nutrition sources for the poor segment of the population. Since many indigenous food plants grow wild, they are accessible, they can be collected freely and are thus available to everyone, including the poor (Kabuye et al. 1999). Fruits and vegetables are of great nutritional value. They are important sources of vitamins and minerals that are essential for human health and well-being. Their consumption ensures the intake of various essential vitamins and mineral elements thus avoiding the problem of malnutrition (Yamaguchi 1983). There is a wide variety of indigenous vegetables and fruits found in Africa, which are chief sources of nutrients, vitamins, antioxidants, minerals, and proteins (Odhav et al. 2007; Ogwu et al. 2016; Ogwu 2020). Some of the indigenous vegetables and fruits are mainly used for medicinal purposes (Eifediyi et al. 2008).

Names and naming are important determinant factors in local society and contributing to promoting sustainable plant utilization and conservation (Penny 2001; Ogwu and Osawaru 2014; Ogwu et al. 2014). Plants are more easily recognized by their local names in every part of the world. These local names play a vital role in ethnobotanical study of a specific tribe or region (Singh 2008). The local names of crop plants, especially in Bini language, are reported among tribes surveyed. Documentation of local names is highly valued by Rogers (1963), Rogers and Applan (1973), Allem (2000), Bressan et al. (2005), Sawadogo et al. (2005), and Osawaru and Dania-Ogbe (2010). Local names are used to promote and trade plant and plant products in all the local markets assessed with little to no reference to their scientific nomenclature. Although local names are not directly recommended for scientific discussions because they lack uniformity and consistency (Singh 2008), yet they may certainly be considered as a useful tool for obtaining useful information on plants. Local names provide means of reference by local people in a particular 
area. Also, in some cases, the plants are well known with their local name than the common names. This is the case of "ebolebo" and "dogoyaro" for Indian almond and neem, respectively.

In conclusion, plants provide valuable functions as foods, raw materials, socioeconomic development, as well as sustainable environmental development and indicator of climate change impacts. They have been used as a means of livelihood sustenance and preservation of indigenous knowledge through their utilization pattern for centuries. This chapter established that local markets are a data bank for economic plant species. The diversity of these plants and plant products in the various local market assessed suggest that despite ongoing climate change, some level of plant production and plant-human interaction is ongoing. This chapter also revealed that urban markets have a higher number of plants and plant products compared to peri-urban and rural markets. This is a reflection that lesser populated markets are less diverse in terms of plants and plant products in the market. Additionally, majority of the plants and plant products are utilized as food with fruits being the predominant part that is used as food. Overall, the vendors/sellers are predominantly women and their interaction with plants in the market makes them an important group in the fight against climate change, food insecurity, and biodiversity crisis.

\section{References}

Agea GJ, Kimondo JM, Okia CK, Abohassan RAA, Obua J, Hall J, Teklehaimanot Z (2011) Contribution of wild and semi wild food plants to overall household diet in Bunyoro Kitara Kingdom. Ugandan Agric J 6(4):134-144

Allem AC (2000) Ethnobotanical testimony on the ancestors of cassava (Manihot esculenta Crantz. subsp. esculenta). Plant Genet Resour Newsl 123:19-22

Atedhor GO, Odjugo PAO, Uriri AE (2011) Changing rainfall and anthropogenic-induced flooding: impacts and adaptation strategies in Benin City, Nigeria. J Geogr Reg Plan 4(1):42-52

Bressan EA, Veasey EA, Peroni N, Felipim AP, Pacheo dos Santos KM (2005) Collecting yam (Dioscorea spp.) and sweet potato (Ipomea batatas) germplasm in traditional agriculture smallholding in the Vale do Riberira, Sao Paulo, Brazil. Plant Genet Resour Newsl 144:8-13

Burkill RM (1985) The useful plants of tropical West Africa, vol 1. Royal Botanic Gardens, Kew. $635 p$

Bye RA, Linares E (1983) The role of plants found in the Mexican markets and their importance in ethnobotanical studies. J Ethnobiol 3:1-13

Cunningham AB (2001) Applied ethnobotany: people, wild plant use and conservation. People and plants conservation manual. Earthscan, London. 300p

De Caluwe E (2011) Market chain analysis of baobab (Adansonia digitata L.) and tamarind (Tamarindus indica L.) products in Mali and Benin. $\mathrm{PhD}$ thesis, Faculty of Bioscience Engineering, Ghent University, Ghent

Efe SI, Eyefia OA (2014) Urban warming in Benin City, Nigeria. Atmos Clim Sci 4:241-252

Eifediyi K, Mensah JK, Ohaju-Obodo JO, Okoli RI (2008) Phytochemical, nutritional and medicinal properties of some leafy vegetables consumed by Edo people of Nigeria. Afr J Biotechnol 7:2304-2309

Floyd AC, Oikpor R, Ekene B (2016) An assessment of climate change in Benin City, Edo State, Nigeria. FUTY J Environ 10(1):87-94 
Gill LS, Nyawuame HGK, Omoigui JD (1993) A survey of economic plants sold in the market of Benin City (Edo State) Nigeria. Niger Technol Educ 10:69-77

Idu M, Osawaru ME, Orhue E (2005) Ethno-medicinal plant products in some local markets on Benin City, Nigeria. Ethnobotany 17:118-122

Idu M, Erhabor JO, Efijuemue HM (2010) Documentation on medicinal plants sold in markets in Abeokuta, Nigeria. Trop J Pharm Res 9(2):110-118

Johns T (2004) Underutilized species and new challenges in global health. LEIZ Mag 20(1):5-6

Johns T, Eyzaguirre PB (2002) Nutrition and the environment. In: Nutrition: a foundation for development, vol 20. ACC/SCN, Geneva, pp 269-285

Johns T, Sthapit BR (2004) Biocultural diversity in the sustainability of developing country food systems. Food Nutr Bull 25(2):143-155

Johnson EJ, Johnson TJ (1976) Economic plants in a rural Nigerian market. Econ Bot 30:375-381

Kabuye CHS, Maundu PM, Ngugi W (1999) Traditional food plants of Kenya. Kenya Resource Centre for Indigenous Knowledge, Nairobi

Keratela YY, Hussain HSH (1990) Indigenous fruits sold in markets of Ishan area of Bendel State. The Nigerian Field 55:12-18

Martin G (1992) Searching for plants in peasant marketplaces. In: Plotkin MJ, Famolare L (eds) Sustainable harvest and marketing of rainforest products. Island Press, Washington, DC, pp 212-223

Mekasha J, Tirfe GG (2019) Assessing factors affecting marketing of vegetable products: the case of Qewet woreda, Ethiopia. IOSR J Bus Manag 21(4):82-93

Muhanji G, Roothaert R, Webo C, Stanley M (2011) African indigenous vegetable enterprise and market access for small-scale farmers in East Africa. Int J Agric Sustain 9(1):194-202

Nguyen MT (2005) Cultivated plant collections from marketplaces. Ethnobot Res Appl 3:5-15

Obiri BD, Addai A (2007) People and plants: a survey of economic botanicals on the Kumasi central market. Ghana J For 21 and 22:50-71

Odhav B, Beekrum S, Akula U, Baijnath H (2007) Preliminary assessment of nutritional value of traditional leafy vegetables in KwaZulu-Natal, South Africa. J Food Compos Anal 20:430-435

Ogwu MC (2019) Towards sustainable development in Africa: the challenge of urbanization and climate change adaptation. In: Cobbinah PB, Addaney M (eds) The geography of climate change adaptation in urban Africa. Springer Nature, Cham. 29-55pp. https://doi.org/10.1007/ 978-3-030-04873-0 2

Ogwu MC (2020) Value of Amaranthus [L.] species in Nigeria. In: Waisundara V (ed) Nutritional Value of Amaranth. IntechOpen, London. 1-21pp. https://doi.org/10.5772/intechopen.86990

Ogwu MC, Osawaru, ME (2014) Comparative Study of Microflora Population on the Phylloplane of Common Okra [Abelmoschus esculentus L. (Moench.)]. Nigerian Journal of Biotechnology 28:17-25

Ogwu MC, Osawaru ME, Ahana CM (2014) Challenges in conserving and utilizing plant genetic resources (PGR). Int J Genet Mol Biol 6(2):16-22. https://doi.org/10.5897/IJGMB2013.0083

Ogwu MC, Osawaru ME, Aiwansoba RO, Iroh RN (2016) Status and prospects of vegetables in Africa. In: Borokini IT, Babalola FD (eds) Conference proceedings of the joint biodiversity conservation conference of Nigeria Tropical Biology Association and Nigeria Chapter of Society for Conservation Biology on MDGs to SDGs: toward sustainable biodiversity conservation in Nigeria. University of Ilorin, Ilorin. $47-57 \mathrm{pp}$

Ogwu MC, Osawaru ME, Obahiagbon GE (2017) Ethnobotanical survey of medicinal plants used for traditional reproductive care by Usen people of Edo State, Nigeria. Malaya J Biosci $4(1): 17-29$

Osawaru ME, Dania-Ogbe FM (2010) Enthnobotanical revelations and traditional uses of West African Okra [Abelmoschus caillei (A. Chev.) Stevels] among tribes in South-Western Nigeria. Plant Arch 10:211-217

Osawaru ME, Odin EI (2012) An inventory of plants and plant products in rural and urban markets in some localities in Southern Edo State, Nigeria. Univ Benin J Sci Technol 1:1-13

Osawaru ME, Ogwu MC (2014a) Conservation and utilization of plant genetic resources. In: Omokhafe K, Odewale J (eds) Proceedings of 38th annual conference of The Genetics Society of Nigeria. Empress Prints Nigeria Limited, Benin City, Nigeria, pp 105-119 
Osawaru ME, Ogwu MC (2014b) Ethnobotany and germplasm collection of two genera of cocoyam (Colocasia [Schott] and Xanthosoma [Schott], Araceae) in Edo State Nigeria. Sci Technol Arts Res J 3(3):23-28. https://doi.org/10.4314/star.v3i3.4

Penny RA (2001) Gender and Indigenous knowledge experiences in Nigeria and the USA. Indig Knowl Dev Monit 9(1):16-17

Rogers DJ (1963) Studies of Manihotesculenta Cranz and related species. Bull Torrey Bot Club 90:43-54

Rogers DJ, Applan SG (1973) Manihot and Manihotoides (Euphorbiaceae), a computer assisted study, Flora Neotropica, monograph no 13. Hofner Press, New York

Romanik C (2008) An urban-rural focus on food markets in Africa. Research report. The Urban Institute. 45p. https://www.urban.org/sites/default/files/publication/31436/411604-An-UrbanRural-Focus-on-Food-Markets-in-Africa.PDF

Sawadogo M, Duedraogo J, Belem Balma M, Dossou B, Jarvis D (2005) Influence of ecosystem component on cultural practices affecting the in situ conservation of agricultural biodiversity. Plant Genet Resour Newsl 141:19-25

Singh H (2008) Importance of local names of some useful plants in ethnobotanical study. Indian J Tradit Knowl 7(2):365-370

Whitaker TW, Cutler HC (1966) Food plants in a Mexican market. Econ Bot 20:6-16

Yamaguchi M (1983) World vegetables, principle, production and nutritive values. Ellishorwood Limited Publishers, Chichester

Yaradua SS, El-Ghani MA (2015) Ethnobotanical survey of edible plants sold in Katsina metropolis markets. Int J Sci Res 4:884-889

Open Access This chapter is licensed under the terms of the Creative Commons Attribution 4.0 International License (http://creativecommons.org/licenses/by/4.0/), which permits use, sharing, adaptation, distribution and reproduction in any medium or format, as long as you give appropriate credit to the original author(s) and the source, provide a link to the Creative Commons license and indicate if changes were made.

The images or other third party material in this chapter are included in the chapter's Creative Commons license, unless indicated otherwise in a credit line to the material. If material is not included in the chapter's Creative Commons license and your intended use is not permitted by statutory regulation or exceeds the permitted use, you will need to obtain permission directly from the copyright holder. 